\title{
Psychological distress and health-related quality of life in patients with bone marrow edema syndrome
}

\author{
Timo Zippelius ${ }^{1}$, Georg Matziolis ${ }^{1}$, Eric Röhner ${ }^{1}$, Christoph Windisch ${ }^{1,2}$, Chris Lindemann $^{1}$, Patrick Strube $^{1}$ \\ ${ }^{1}$ Orthopaedic Department of the Waldkliniken Eisenberg, Orthopaedic Professorship of the University Hospital Jena, Eisenberg, Germany; \\ ${ }^{2}$ Orthopaedic Department, Helios Klinik Blankenhain, Blankenhain, Germany \\ Contributions: (I) Conception and design: T Zippelius, G Matziolis, P Strube; (II) Administrative support: G Matziolis, C Windisch; (III) Provision of \\ study materials or patients: E Röhner, C Lindemann; (IV) Collection and assembly of data: E Röhner, C Windisch, C Lindemann; (V) Data analysis \\ and interpretation: G Matziolis, P Strube; (VI) Manuscript writing: All authors; (VII) Final approval of manuscript: All authors. \\ Correspondence to: Timo Zippelius, Dr. med. Orthopaedic Department of the Waldkliniken Eisenberg, Orthopaedic Professorship of the University \\ Hospital Jena, Klosterlausnitzer Str. 81, 07607 Eisenberg, Germany. Email: timo.zippelius@uni-jena.de.
}

Background: The aim of the study was to investigate psychological distress and health-related quality of life (HrQoL) in patients with bone marrow edema syndrome (BMES) of the hip or knee joint.

Methods: This retrospective study included patients with the diagnosis BMES treated in the period 2016-2017. As well as analyzing the epidemiological data (age, sex, vascular disease, hypertension, etc.), we used the Hospital Anxiety and Depression Scale (HADS) to document anxiety and depression and the fivelevel version of the EuroQol Group's EQ-5D instrument (EQ-5D-5L) to assess HrQoL and compared it to historical controls of the healthy population.

Results: The study group comprised 56 patients (26 females, 30 males) with a mean age of 55.8 (range, 15-84) years. HADS: there was no difference between the study and control cohorts in the rates of anxiety $(\mathrm{P}=0.595)$ or depression $(\mathrm{P}=0.241)$. EQ-5D-5L: the HrQoL was significantly lower in the patients with BMES than in the healthy controls both for parameters of the EQ-5D-5L index and in the various age groups. No difference in HrQoL was seen between BMES of the hip and the knee or among the different radiological stages of BMES.

Conclusions: The patients with BMES displayed a clear reduction in HrQoL, but this was not associated with psychological distress with regard to significant anxiety and depression. Patients with BMES and a high score for anxiety and depression are at great risk of chronic pain, and we recommend they should receive psychological counseling.

Keywords: Anxiety and depression; bone marrow edema syndrome (BMES); health-related quality of life (HrQoL); psychological distress

Submitted Jul 07, 2019. Accepted for publication Sep 10, 2019.

doi: 10.21037/atm.2019.09.115

View this article at: http://dx.doi.org/10.21037/atm.2019.09.115

\section{Introduction}

Bone marrow edema syndrome (BMES) is a painful disease with pathological increase of the interstitial fluid in bone, usually affecting a joint (1). BMES occurs preferentially in the lower extremity in men (3:1) aged 20-40 years (2). Because BMES is often diagnosed late and attempts at treatment are frequently unsuccessful, $80 \%$ of patients develop osteonecrosis (ON) 3-18 months after onset. In some cases, this even necessitates joint replacement (3-8). $\mathrm{ON}$ is a global health-care burden. According to this, there are 15,000 to 20,000 new cases of $\mathrm{ON}$ each year in the USA alone and studies showed that the average prevalence was 28.91 per 100,000 populations in Korea (9). In 2004 the number of patients who sought medical care for $\mathrm{ON}$ in Japan was about 11,400 (10). Besides this, Bergman et al. showed that the incidence rate was 4.7 cases per 10,000 
among patients 50 years and older in Sweden in 2005 (11). Additionally, in the United Kingdom the incidence of ON ranges from about 1.4 to 3.0/100,000 including data from approximately 3.4 million active patients (12). On top of this, Zhao et al. estimated that there are 8.12 million cases with $\mathrm{ON}$ of the femoral head among Chinese people aged 15 years and older (13). It is thought that $10-12 \%$ of all total hip replacements are due to $\mathrm{ON}(2,14-16)$.

Chen et al. recently determined that female patients with $\mathrm{ON}$ in the proximal femur are at a higher risk of anxiety and depression (17). Anxiety and depression limit the ability to work, worsen the health-related quality of life (HrQoL), and thus lead to socio-economic problems (18). It has not yet been established whether psychological distress (anxiety and depression) plays a part in the genesis of $\mathrm{ON}$ or is already present in the early stages of ON (i.e., BMES).

The primary aim of the present study was therefore to investigate psychological distress and self-reported HrQoL in patients with BMES. The hypothesis was that patients with BMES that has not yet progressed to $\mathrm{ON}$ are more likely to suffer anxiety or depression than the normal population and also have poorer HrQoL. A secondary goal was to find out whether HrQoL or psychological distress is affected by the stage or site of the disease.

\section{Methods}

\section{Patients and study design}

This retrospective study was conducted at the University Hospital Jena/Orthopaedic Department, Campus Eisenberg, Thuringia, Germany and included patients with the diagnosis of BMES of the proximal femur or the knee joint who were treated in our hospital in 2016 or 2017. Those whose treatment was less than 12 months ago were excluded. The selection criterion in screening the electronic medical records was diagnosis M87.* according to the International Statistical Classification of Diseases and Related Health Problems (ICD-10-GM, German Modification).

Patients with previous joint replacement surgery were excluded.

\section{Standard treatment of patients with BMES}

Following a standard treatment scheme $(3,19)$, all patients were admitted to the hospital and received a 5-day course of intravenous Iloprost (Ilomedin, Bayer Schering, Germany).
Patients with Association Research Circulation Osseous (ARCO) stage $\geq 2$ were additionally treated with surgical decompression beforehand (4). In all patients only partial loading of the affected joint (max. $15 \mathrm{~kg}$ weight bearing using two forearm crutches) was permitted for 6 weeks after treatment.

\section{Data acquisition}

All patients were given a questionnaire for self-reporting of psychological distress and HrQoL. The Hospital Anxiety and Depression Scale (HADS) was used to document anxiety and depression and the five-level version of the EuroQol Group's EQ-5D instrument (EQ-5D-5L) to assess HrQoL. The established cut-offs described in the literature were employed to categorize patients as suffering from anxiety or depression ( $\geq 11$ for anxiety and $\geq 9$ for depression) (20). The EQ-5D-5L index was used to evaluate the EQ-5D$5 \mathrm{~L}$ results. A mean value of 0.91 (health status of the South Australian population) was taken as reference for the normal population and corresponds approximately to the health status of the local population (21). Moreover, the visual analog scale of the EQ-5D-5L (EQ-VAS) was used. Ratings on this scale of 0 to $100 \mathrm{~mm}$ represent a global evaluation of the self-perceived health status, where 0 stands for the worst imaginable and 100 for the best imaginable state of health (21-24).

The data published by Hinz et al. in 2011 (25) (for more than 4,400 persons) and McCaffrey et al. in 2016 (24) (more than 2,900 persons), also acquired by means of HADS und EQ-5D-5L (EQ-VAS), served as reference/control group for comparison.

Furthermore, we documented the following parameters: radiological stage of BMES according to ARCO, based on radiography and preinterventional magnetic resonance imaging (MRI) (26); site of edema (hip or knee); presence of vascular disease, hypertension, diabetes mellitus, or lipid metabolism disorders; nicotine or alcohol consumption (yes/ no); sex (female/male); body mass index (BMI); age group (15-24, 25-34, 35-44, 45-54, 55-64, 65-74, 75+ years).

\section{Data analysis}

With regard to the primary hypothesis, psychological distress was evaluated by comparing the rates of anxiety and depression in the study group and the control cohort. Based on the parameters EQ-VAS and EQ-5D-5L index, the effect of the disease on $\mathrm{HrQoL}$ was compared by age group 
and sex between patients with BMES and the control group.

Secondarily, we analyzed the influence of disease stage and site of edema (hip or knee joint) on the results of HADS and EQ-5D-5L in the BMES group.

\section{Statistical analysis}

Statistical analysis was performed with GraphPad Prism V.5.0a (GraphPad Software Inc., San Diego, USA) and SPSS V.24 (IBM, Armonk, USA). Continuous parameters were tested with a two-sided, two-way ANOVA, or a twosided unpaired $t$-test, categorical parameters with Fisher's exact test. The level of significance was set to $\mathrm{P}=0.05$.

\section{Results}

\section{Demographic characteristics}

Of the 104 patients with the diagnosis of BMES who were identified and contacted in writing, 56 (53.8\%; 26 females, 30 males) completed and returned the questionnaires. Their mean age was 55.8 years (range, 15-84 years). Further demographic and patient-specific data can be found in Table 1 .

\section{Anxiety and depression}

There was no significant difference between the study group and the control cohort in the rate of either anxiety $(\mathrm{P}=0.595)$ or depression $(\mathrm{P}=0.241$; Table 2$)$.

\section{HrQoL}

Compared with the healthy controls, the patients with BMES had significantly poorer HrQoL on both parameters (EQ-5D-5L index: $\mathrm{P}_{\text {BMES }}<0.001$; EQ-VAS: $\mathrm{P}_{\text {BMES }}=0.001$; Figures 1,2). The age group also had an effect on HrQoL (EQ-5D-5L index: $\mathrm{P}_{\text {age }}=0.036$; EQ-VAS: $\left.\mathrm{P}_{\text {age }}=0.040\right)$, but showed no interaction with that of the disease (EQ-5D-5L index: $\mathrm{P}_{\text {BMES } \times \text { age }}=0.816$; EQ-VAS: $\left.\mathrm{P}_{\text {BMES } \times \text { age }}=0.542\right)$.The patient's sex exerted no influence on the results (EQ-5D$5 \mathrm{~L}$ index: $\mathrm{P}_{\text {gender }}=0.755, \mathrm{P}_{\mathrm{BMES} \times \text { gender }}=0.162 ; \mathrm{EQ}-\mathrm{VAS}: \mathrm{P}_{\text {gender }}$ $\left.=0.416, \mathrm{P}_{\mathrm{BMES} \times \text { gender }}=0.602\right)$.

\section{Influence of site and stage of disease}

The influence of the site of edema and the ARCO stage on the HADS results is shown in Tables 3,4.

Neither the joint affected by edema nor the radiological disease stage had a significant effect on HrQoL in terms of EQ-5D-5L index $\left(\mathrm{P}_{\mathrm{ARCO}}=0.900, \mathrm{P}_{\text {joint }}=0.738, \mathrm{P}_{\mathrm{ARCO}} \times\right.$ joint $=0.592 ;$ Figure 3$)$ or EQ-VAS $\left(\mathrm{P}_{\mathrm{ARCO}}=0.501, \mathrm{P}_{\text {joint }}=0.483\right.$, $\mathrm{P}_{\mathrm{ARCO} \times \text { joint }}=0.383 ;$ Figure 4$)$.

\section{Discussion}

The aim of the present study was to determine the psychological distress and the self-reported HrQoL in patients with BMES compared with the normal population. The secondary goal was to investigate the influence of disease stage and edema site on the outcome parameters. To the best of our knowledge we are the first to demonstrate the presence of a marked worsening of HrQoL, irrespective of age or sex, in BMES, the precursor of ON. In contrast to our hypothesis, however, the BMES patients did not show elevated rates of anxiety and depression. There was also no difference with regard to the joint involved or the radiological stage of BMES.

Earlier studies demonstrated that BMES causes pain and functional impairment (19). Our study confirms these findings in relation to the worsening of self-reported HrQoL owing to pain and functional impairment. Strikingly, the worsening of $\mathrm{HrQoL}$ was evident despite the elapse of at least 1 year since treatment. One explanation for this may be the fact that in many cases the detection and treatment of BMES took several weeks or even months. This can be associated with a degree of chronification of pain in the patients concerned. The International Association for the Study of Pain (IASP) defines chronic pain as "pain that has persisted beyond the normal tissue healing time (usually taken to be 3 months) (27). Thus, the typical evolution of BMES almost inevitably leads to chronic pain, which is then reflected in the HrQoL.

The prevalence of anxiety and depression in the patients with BMES was much lower than in the patients with ON described by Chen et al. (17). While the rates of anxiety (7.1\%; 4/56 patients) and depression (16.1\%; 9/56 patients) in BMES patients do not differ essentially from those of the normal population, the published prevalence in patients with ON seem to be much higher (anxiety: 20.4\%, 44/216 patients; depression: $21.8 \%, 47 / 216$ patients). It can be assumed that, due to the short duration of the disease, a chronic pain disorder has not yet developed and for this reason anxiety and/or depression have not yet progressed. It is well known from other diseases like low back pain that anxiety and depression are strongly linked to the chronicity of pain $(27,28)$. Therefore, either anxiety or 
Table 1 Epidemiologic data of the patients with BMES

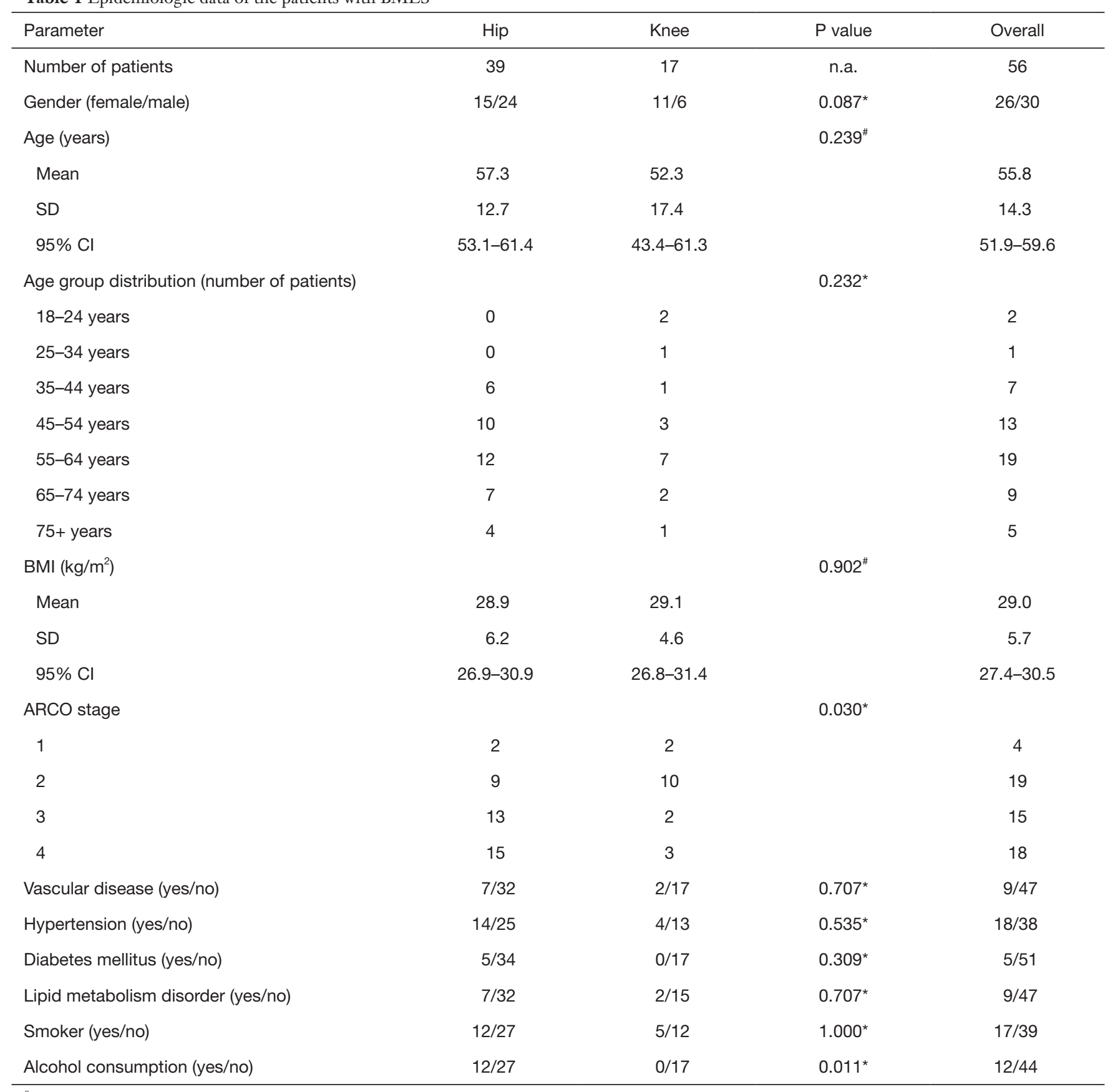

", P values are from unpaired $t$-test; ${ }^{\text {, }} \mathrm{P}$ values are from Fisher's exact test. BMES, bone marrow edema syndrome; ARCO, Association Research Circulation Osseous; SD, single standard deviation; $95 \% \mathrm{Cl}, 95 \%$ confidence interval; n.a., not available.

depression increase with the progression of the disease and the development of ON, or BMES is particularly likely to progress to $\mathrm{ON}$ in a subgroup of patients prone to anxiety and depression. Thus, one can conclude from the data that anxiety and depression are probably not causal factors for
BMES, but it remains open whether anxiety, depression, and psychological distress caused by chronic pain, for example, can trigger progress to $\mathrm{ON}$ or whether they are a consequence of the disease. The results of a small-animal study by Henneicke et al. suggest the former. Using a mouse 
Table 2 Comparison of the results of HADS between BMES patients and normal population

\begin{tabular}{lccc}
\hline \multirow{2}{*}{ HADS category } & \multicolumn{2}{c}{ Group } & P value $^{\#}$ \\
\cline { 2 - 3 } & BMES & Control $^{*}$ & \\
\hline Anxiety & & & 0.595 \\
Anxious & 4 & 208 & \\
Not anxious & 52 & 4,202 & \\
Depression & & & 0.241 \\
Depressive & 9 & 1,044 & \\
Not depressive & 47 & 3,366 & \\
\hline
\end{tabular}

*, data from control group was derived from McCaffrey et al. 2016 (24); \#, P values are from Fisher's exact test. BMES, bone marrow edema syndrome; HADS, Hospital Anxiety and Depression Scale.

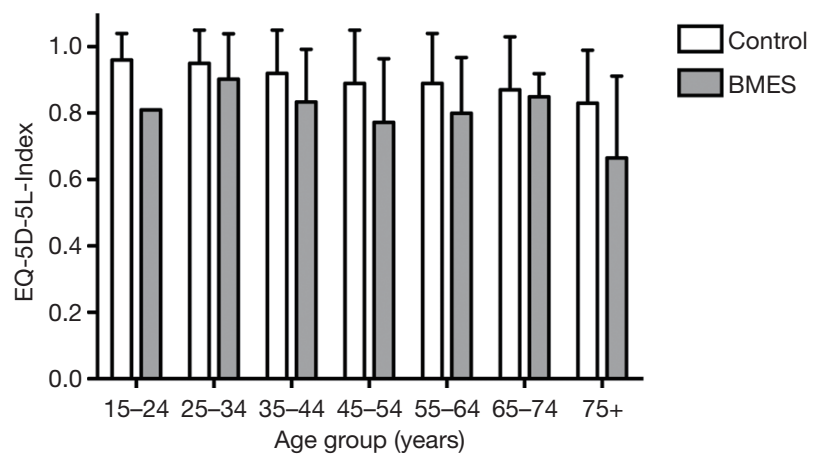

Figure 1 The age group distribution of $\mathrm{HrQoL}$ based on the EQ$5 \mathrm{D}-5 \mathrm{~L}$ index of patients with BMES and the healthy population (24). The influence of age group $(\mathrm{P}=0.036)$ and presence of BMES $(\mathrm{P}<0.001)$, but not their interaction $(\mathrm{P}=0.816)$, had a significant influence on the results. Whiskers represent single standard deviation. HrQoL, health-related quality of life; EQ-5D-5L, the five-level version of the EuroQol Group's EQ-5D instrument; BMES, bone marrow edema syndrome.

model, they found that chronic stress triggered an increase in serum glucocorticoid concentrations, which in turn led to increased osteoclast activity with elevation of bone resorption (29). In a recently published review, Harth and Nielson summarized data demonstrating how inflammatory joint diseases such as osteoarthritis are greatly intensified by the psychological distress resulting from chronic pain (30).

Although other risk factors such as elevated serum lipids or excessive biomechanical loading in obese patients seem to be primarily responsible for the pathogenesis of BMES (31),

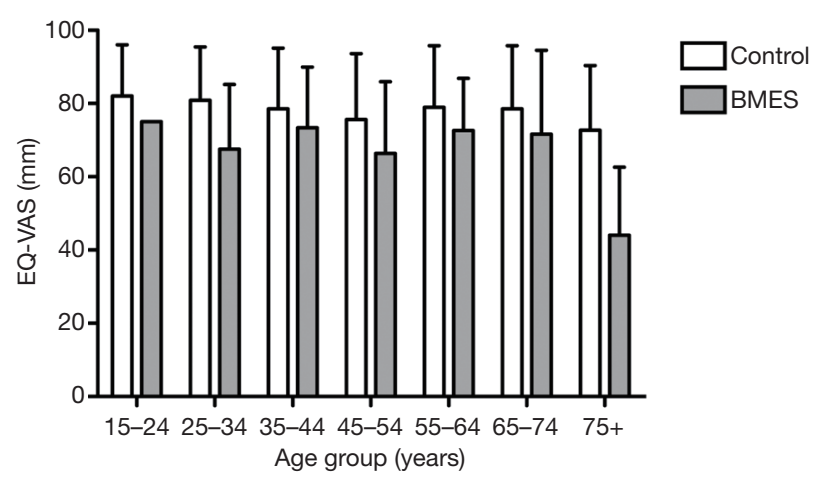

Figure 2 The age group distribution of HrQoL based on the EQVAS of patients with BMES and the healthy population (24). The influence of age group $(\mathrm{P}=0.040)$ and presence of BMES $(\mathrm{P}=0.001)$, but not their interaction $(\mathrm{P}=0.542)$, had a significant influence on the results. Whiskers represent single standard deviation. HrQoL, health-related quality of life; EQ-VAS, the visual analog scale of the EQ-5D-5L; EQ-5D-5L, the five-level version of the EuroQol Group's EQ-5D instrument; BMES, bone marrow edema syndrome.

associations have nevertheless been demonstrated between the accepted risk factors, e.g., obesity, and psychological distress (32). However, it appears that this tends to play a relevant part in the progression to $\mathrm{ON}$ only with increasing duration of disease.

Our study is not without limitations. First, because the numbers of patients with anxiety or depression are relatively small, the power may be insufficient to document a corresponding difference, especially in the subgroups for ARCO stages or individual joints. Second, owing to the study design one can only speculate regarding the further progression of individual patients towards ON. Third, this was a retrospective study at an isolated point in time. No conclusions can be drawn concerning the course of the disease over time. Finally, the control cohort is from a different population (South Australia), so bias cannot be excluded with certainty. Nevertheless, the mean EQ$5 \mathrm{D}-5 \mathrm{~L}$ index, for example, is almost identical in the two populations (South Australia vs. Germany) (21).

\section{Conclusions}

In conclusion, it can be stated that although patients with BMES already demonstrate with decreased HrQoL, this is not (yet) expressed in psychological distress (clinically significant anxiety and depression). Based on the knowledge 
Table 3 Comparison of the results of HADS between the different ARCO stages

\begin{tabular}{|c|c|c|c|c|c|}
\hline HADS category & ARCO 1 & ARCO 2 & ARCO 3 & ARCO 4 & $P$ value \\
\hline Anxious & 0 & 21 & 1 & 0 & \\
\hline Not anxious & 4 & 17 & 14 & 17 & \\
\hline Depression & & & & & 0.839 \\
\hline Not depressive & 3 & 17 & 12 & 15 & \\
\hline
\end{tabular}

*, P values are from Fisher's exact test. HADS, Hospitality Anxiety and Depression Scale; ARCO, Association Research Circulation Osseous.

Table 4 Comparison of the results of HADS between hip and knee joints

\begin{tabular}{lccc}
\hline HADS category & Hip joint & Knee joint & P value* \\
\hline Anxiety & 2 & & 0.577 \\
Anxious & 37 & 15 & 0.707 \\
Not anxious & & & 2 \\
Depression & 7 & 15 & \\
Depressive & 32 & & \\
Not depressive & & & \\
\hline
\end{tabular}

*, P values are from Fisher's exact test. HADS, Hospital Anxiety and Depression Scale.

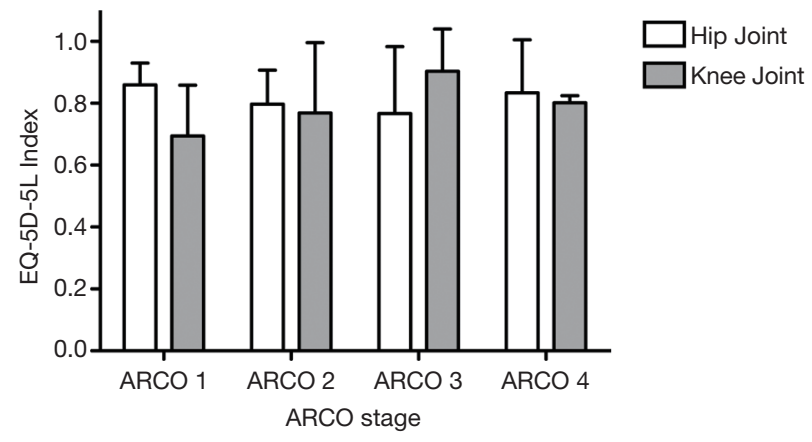

Figure 3 The ARCO stage distribution of HrQoL based on the EQ-5D-5L index of patients with BMES of the hip or knee joint. Neither ARCO stage $(\mathrm{P}=0.900)$, the site of BMES $(\mathrm{P}=0.738)$, nor their interaction $(\mathrm{P}=0.592)$ had a significant influence on the results. Whiskers represent single standard deviation. ARCO, Association Research Circulation Osseous; HrQoL, healthrelated quality of life; EQ-5D-5L, the five-level version of the EuroQol Group's EQ-5D instrument; BMES, bone marrow edema syndrome.

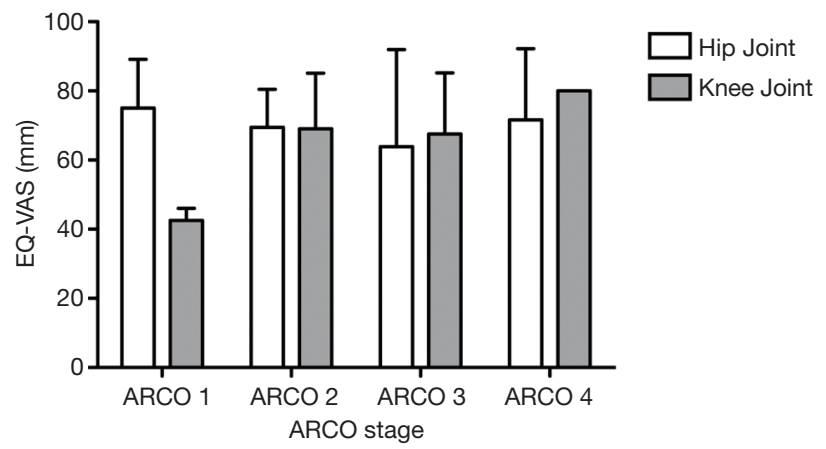

Figure 4 The ARCO stage distribution of HrQoL based on the EQ-VAS of patients with BMES of the hip or knee joint. Neither ARCO stage $(\mathrm{P}=0.501)$, the site of BMES $(\mathrm{P}=0.483)$, nor their interaction $(\mathrm{P}=0.383)$ had a significant influence on the results. Whiskers represent single standard deviation. ARCO, Association Research Circulation Osseous; HrQoL, health-related quality of life; EQ-VAS, the visual analog scale of the EQ-5D-5L; EQ5D-5L, the five-level version of the EuroQol Group's EQ-5D instrument; BMES, bone marrow edema syndrome. 
of increased prevalence of anxiety and depression in patients with $\mathrm{ON}$ and the high likelihood of development of a chronic pain syndrome in BMES, patients with BMES, particularly those who score highly for anxiety and depression, should receive psychological counseling alongside their standard treatment.

\section{Acknowledgments}

None.

\section{Footnote}

Conflicts of Interest: The authors have no conflicts of interest to declare.

Ethical Statement: The authors are accountable for all aspects of the work in ensuring that questions related to the accuracy or integrity of any part of the work are appropriately investigated and resolved. The study was approved by the local ethics committee under rule number 5494-04/18/18.

\section{References}

1. Geith T, Niethammer T, Milz S, et al. Transient Bone Marrow Edema Syndrome versus Osteonecrosis: Perfusion Patterns at Dynamic Contrast-enhanced MR Imaging with High Temporal Resolution Can Allow Differentiation. Radiology 2017;283:478-85.

2. Pountos I, Giannoudis PV. The role of Iloprost on bone edema and osteonecrosis: Safety and clinical results. Expert Opin Drug Saf 2018;17:225-33.

3. Röhner E, Zippelius T, Steindl D, et al. Effects of intravenous iloprost therapy in patients with bone marrow oedema of the foot and ankle. Eur J Orthop Surg Traumatol 2014;24:1609-16.

4. Maus U, Roth A, Tingart M, et al. S3 Guideline. Part 3: Non-Traumatic Avascular Necrosis in Adults - Surgical Treatment of Atraumatic Avascular Femoral Head Necrosis in Adults. Z Orthop Unfall 2015;153:498-507.

5. Vande Berg B, Lecouvet F, Koutaissoff S, et al. Bone marrow edema of the femoral head. JBR-BTR 2007;90:350-7.

6. Elder GJ. From marrow oedema to osteonecrosis: common paths in the development of post-transplant bone pain. Nephrology (Carlton) 2006;11:560-7.

7. Meizer R, Radda C, Stolz G, et al. MRI-controlled analysis of 104 patients with painful bone marrow edema in different joint localizations treated with the prostacyclin analogue iloprost. Wien Klin Wochenschr 2005;117:278-86.

8. Symptomatic multifocal osteonecrosis. A multicenter study. Collaborative Osteonecrosis Group. Clin Orthop Relat Res 1999;(369):312-26.

9. Kang JS, Park S, Song JH, et al. Prevalence of osteonecrosis of the femoral head: a nationwide epidemiologic analysis in Korea. J Arthroplasty 2009;24:1178-83.

10. Fukushima W, Fujioka M, Kubo T, et al. Nationwide epidemiologic survey of idiopathic osteonecrosis of the femoral head. Clin Orthop Relat Res 2010;468:2715-24.

11. Bergman J, Nordström A, Nordström P. Epidemiology of osteonecrosis among older adults in Sweden. Osteoporos Int 2019;30:965-73.

12. Cooper C, Steinbuch M, Stevenson R, et al. The epidemiology of osteonecrosis: findings from the GPRD and THIN databases in the UK. Osteoporos Int 2010;21:569-77.

13. Zhao DW, Yu M, Hu K, et al. Prevalence of Nontraumatic Osteonecrosis of the Femoral Head and its Associated Risk Factors in the Chinese Population: Results from a Nationally Representative Survey. Chin Med J (Engl) 2015;128:2843-50.

14. Lavernia CJ, Sierra RJ, Grieco FR. Osteonecrosis of the femoral head. J Am Acad Orthop Surg 1999;7:250-61.

15. Steinberg ME, Hayken GD, Steinberg DR. A quantitative system for staging avascular necrosis. J Bone Joint Surg Br 1995;77:34-41.

16. Mont MA, Hungerford DS. Non-traumatic avascular necrosis of the femoral head. J Bone Joint Surg Am 1995;77:459-74.

17. Chen SB, Hu H, Gao YS, et al. Prevalence of clinical anxiety, clinical depression and associated risk factors in chinese young and middle-aged patients with osteonecrosis of the femoral head. PLoS One 2015;10:e0120234.

18. Chisholm D, Sweeny K, Sheehan P, et al. Scaling-up treatment of depression and anxiety: a global return on investment analysis. Lancet Psychiatry 2016;3:415-24.

19. Zippelius T, Perka C, Preininger B, et al. Long-term effects of intravenous iloprost therapy in patients with bone marrow oedema of the hip. Acta Orthop Belg 2018;84:172-8.

20. Bjelland I, Dahl AA, Haug TT, et al. The validity of the Hospital Anxiety and Depression Scale. An updated literature review. J Psychosom Res 2002;52:69-77.

21. Hinz A, Kohlmann T, Stöbel-Richter Y, et al. The quality 
of life questionnaire EQ-5D-5L: psychometric properties and normative values for the general German population. Qual Life Res 2014;23:443-7.

22. Janssen MF, Bonsel GJ, Luo N. Is EQ-5D-5L Better Than EQ-5D-3L? A Head-to-Head Comparison of Descriptive Systems and Value Sets from Seven Countries. Pharmacoeconomics 2018;36:675-97.

23. Janssen MF, Szende A, Cabases J, et al. Population norms for the EQ-5D-3L: a cross-country analysis of population surveys for 20 countries. Eur J Health Econ 2019;20:205-16.

24. McCaffrey N, Kaambwa B, Currow DC, et al. Healthrelated quality of life measured using the EQ-5D-5L: South Australian population norms. Health Qual Life Outcomes 2016;14:133.

25. Hinz A, Brähler E. Normative values for the hospital anxiety and depression scale (HADS) in the general German population. J Psychosom Res 2011;71:74-8.

26. Schmitt-Sody M, Kirchhoff C, Mayer W, et al. Avascular necrosis of the femoral head: inter- and intraobserver variations of Ficat and ARCO classifications. Int Orthop

Cite this article as: Zippelius T, Matziolis G, Röhner E, Windisch C, Lindemann C, Strube P. Psychological distress and health-related quality of life in patients with bone marrow edema syndrome. Ann Transl Med 2019;7(20):552. doi: 10.21037/ atm.2019.09.115
2008;32:283-7.

27. Classification of chronic pain. Descriptions of chronic pain syndromes and definitions of pain terms. Prepared by the International Association for the Study of Pain, Subcommittee on Taxonomy. Pain Suppl 1986;3:S1-226.

28. Verhaak PF, Kerssens JJ, Dekker J, et al. Prevalence of chronic benign pain disorder among adults: a review of the literature. Pain 1998;77:231-9.

29. Henneicke H, Li J, Kim S, et al. Chronic Mild Stress Causes Bone Loss via an Osteoblast-Specific Glucocorticoid-Dependent Mechanism. Endocrinology 2017;158:1939-50.

30. Harth M, Nielson WR. Pain and affective distress in arthritis: relationship to immunity and inflammation. Expert Rev Clin Immunol 2019;15:541-52.

31. Lim YZ, Wang Y, Wluka AE, et al. Association of obesity and systemic factors with bone marrow lesions at the knee: a systematic review. Semin Arthritis Rheum 2014;43:600-12.

32. Sinha R, Jastreboff AM. Stress as a common risk factor for obesity and addiction. Biol Psychiatry 2013;73:827-35. 